\title{
A Distributed Multi-Agent Framework for Simulating the Diffusion of Innovations*
}

\author{
Serban GEORGESCU** and Hiroshi OKUDA*** \\ ** The University of Tokyo, Department of Quantum Engineering and Systems Science \\ Bunkyo-ku, Tokyo 113-8654, Japan \\ E-mail: serban@nihonbashi.race.u-tokyo.ac.jp \\ *** Research into Artifacts, Center for Engineering, The University of Tokyo \\ Kashiwanoha, Chiba 277-8568, Japan \\ E-mail: okuda@ race.u-tokyo.ac.jp
}

\begin{abstract}
In this paper we report on the newly developed framework MADS (Multi-Agent Diffusion Simulation), designed for simulating the diffusion of innovations. Created for but not limited to the simulation of the diffusion of emergent technologies, MADS is both close to the diffusion theory and, by using a distributed agent infrastructure, performs data management and streamlines the simulation process. After presenting the framework, we show how it was applied to the simulation of the diffusion of fuel cell vehicles. Unlike the traditional simulation methods usually employed in this case, real consumer preferences are included and a sensitivity analysis is performed.
\end{abstract}

Key words : Innovation Diffusion; Distributed Simulation; Multi-Agent Simulation;

\section{Introduction}

Nowadays, various green technologies exist, among the best known being fuel cell vehicles and household cogeneration systems. However, these products are either in the prototype stage, or for various reasons (e.g. reduced efficiency, uncertainty regarding viability) diffuse very slowly into the market. Due to the increasing pressure in diminishing $\mathrm{CO}_{2}$ emissions, a fast diffusion of these technologies (in particular the spread of hydrogen powered vehicles) is most needed. In the view of understanding the underlying phenomena of this diffusion process as well as discovering ways in which it can be enhanced, computer simulations are the most efficient approach.

Extensive work has been done to create tools for energy - technology - environmental systems modeling. The de facto standard software tools for this kind of modeling are the MARKAL $^{(1)}$, NEMS $^{(2)}$ and MESSAGE-MACRO ${ }^{(3)}$ systems. Being provided input data in form of scenarios, they find a least cost set of technologies that satisfies end-use energy service demands and user-specified constraints. Most of today's forecasts are created in this way; because of this, throughout this paper we will refer to this method as the traditional approach.

In this traditional approach, a perfectly rational consumer chooses the least expensive alternative from a list of available products or technologies; the price is computed by combining efficiency parameters (e.g. fuel efficiency and emissions, in the case of a vehicle) and energy prices (computed by going backwards in the energy network). This transforms the simulation into a linear optimization problem, which is solved using specialized solvers. The strong points of this simulation methodology are the following: it is deterministic, fast and fairly accurate. The accuracy comes from considering the whole economic system and not just an isolated component.

Unfortunately, there is always a price to be paid. The cost-minimization based deterministic model, which is what makes the optimization approach possible, has some significant drawbacks as well. Cost and efficiency are not the only things that motivate a consumer to 
choose a particular product. Other attributes like acceleration, speed, and fuel availability, in the case of vehicles, matter as well. Different consumers usually assign them different weights. Decisions are also influenced by the consumers' entourage. To conclude, there are a lot of factors not accounted for in such simulations.

In trying to complement the traditional optimization-based modeling approach, various studies began to investigate green technology adoption from an innovation diffusion perspective $\mathrm{e}^{(4)-(8)}$. In contrast with the traditional method, where an entire economic sector or the whole economy are being simulated, here the focus is on only a particular product or technology. On the flip side, one can now define a much more complex utility function which can include sensitivity to various attributes, consumer heterogeneity, social influence and so on, usually computed by valuating these preferences and computing an actualized price. On the other hand, one cannot account for externalities or for any kind of feedback; the needed values, usually obtained by traditional simulation, must be taken as independent trends. Consequently, innovation-diffusion based simulations cannot replace traditional ones. However, they can function as a complement by providing sensitivity analysis with respect to aspects not considered in the traditional approach.

Aiming at a better understanding of the underlying phenomena, we have been investigating the possibility of simulating factors affecting the transition to the Hydrogen Society, a futuristic society where hydrogen is used as the main energy carrier. As the leading factor of this transition, we concentrated mainly on studying the adoption of fuel cell vehicles (FCVs). As from a well to wheel point of view FCVs are only low carbon if the hydrogen is actually produced from renewable sources, we considered that with increasing demand, with the advance of cheaper hydrogen production techniques and due to tax regulations, the hydrogen supply will be mostly produced as such. Our attention was pointed towards the consumerside, e.g. how do consumer preferences and consumer-consumer interaction influence the FCV adoption path. We therefore approached this problem from an innovation-diffusion perspective. With time, it was realized that such a simulation must account for many variables, for which data has to be gathered, shared, and updated on a regular basis. As simulations became more complex, it became clear that a specialized simulation software was necessary. Since to the best of our knowledge no such software was available, we began implementing a new one. This is how the MADS ${ }^{(9)}$ project began. The platform is currently in active development and it's open source.

As a multi-agent diffusion simulation framework, MADS is by no means alone. Various such frameworks have been developed, some of the most famous being SWARM ${ }^{(10)}$, $\operatorname{REPAST}^{(11)}, \mathrm{JADE}^{(12)}$ or MADKIT ${ }^{(13)}$. One can use these tools and many other to simulate any diffusion process but, as these are general simulation tools, one has to build everything from the ground up. Multi-agent systems built with economic simulation in mind, like $\operatorname{ASCAPE}^{(14)}, \operatorname{REPAST}^{(11)}$, CORMAS $^{(15)}$ or MODULECO ${ }^{(16)}$ are not the perfect solution for the innovation diffusion problem either, as they are too concentrated on simulating trade and the interaction of complex economic entities or on defining complex behavioral patterns. In our view, for innovation-diffusion simulations, being close to the theory and expanding the data/model management features is the way to go. Extremely complex marketing strategies or consumer behaviors are not so useful, as meaningful data to support such models is almost impossible to get.

MADS uses a distributed infrastructure, also supported by agents, for data and model management. In itself, this is not a new idea. Other distributed simulation systems, like D'AGENTS ${ }^{(17)}$, JINI ${ }^{(18)}$, GALATEA ${ }^{(19)}$ and so on have been implemented. However, these systems are either focused on accessing remote real-time data sources or managing data access privileges or use unfamiliar programming or scripting language or design paradigms. Other alternatives, most notable the HLA (High Level Architecture) are also not straightforward enough to use for the average researcher who either lacks the necessary programming skills or simply wants to contentrate on the science rather than of the implementation. We believe 
the distributed data management system implemented in MADS, formed by running serviceproviding agents, at remote locations, communicating seamlessly with the simulation and providing it with data and various computational services, to be unique to this framework.

Regarding our target, MADS would benefit a wide range of researchers, as besides the field of environmentally related emergent technologies, the study of the diffusion of new products and technologies is very important both for marketing and socio-economic analysis. Although the current focus of the paper is on the diffusion of FCVs, the usability of the framework is by no means limited to vehicle simulations.

Some of most important advantages of using MADS are:

- writing simulations is much easier and intuitive at a high abstraction level (in terms of consumers, suppliers, products and so on) than it would be in terms of objects and vectors

- the structure of the framework imposes a structure on the simulation design thus organizing the simulation creating process

- the distributed model/data management system cuts back on the lengthy data acquisition process by enabling easy sharing

- there is only one central place one has to update models or data

The focus of this paper is two-fold. Our long term goal is the analysis of the diffusion of green products and technologies, in particular the ones related to the Hydrogen Society, hence part of this paper will be dedicated to this type analysis, concentrated on the diffusion of FCVs. As a first step towards this goal, we have developed the MADS framework as a tool to make such analysis easier, therefore the rest of the paper will focus on describing the underlying concepts and the general usage of this tool.

This paper is structured in the following way: In section 2.1, we describe the main models present in the innovation diffusion literature; we then extract patterns and commonalities in section 2.2. Based on these simulation patterns, we develop the distributed infrastructure, as described in section 3.1. We present the simulation design flow in section 3.2, flow which we apply to the FCV diffusion, in section 4.1. The results are shown and discussed in section 4.2. Finally, conclusions are drawn in section 5.

In the course of this paper we will be referring to agents. This word will be used here with two meanings, depending on context. When speaking about MADS infrastructure, agents represent autonomous programs, connected to the MADS server, that communicate with and provide services to the main simulation. When speaking about the diffusion process, agents will represent the actors (households, groups of similar households) that participate (decide) in the process of adoption.

\section{Models \& Patterns}

\subsection{Diffusion models}

We investigated the most widely accepted and used models from the innovation diffusion literature. We then selected the ones to be included in the framework on two criteria: they should be suitable for simulating the diffusion of emergent technologies and complex enough to require the support of a framework.

As a first step, we identified micro (actor level) and macro (aggregated) models. Macro models need to be fitted with historical data, which is unavailable in the case of emergent technologies. They are also simple enough to be easily implemented in any software. We therefore did not consider these models and concentrate only on the micro case.

In the micro category, we differentiate between models which consider direct interaction between agents (interaction-based) and models which consider no or just indirect interaction (non-interaction-based).

In the non-interaction-based class we find equilibrium diffusion models ${ }^{(20)}$ which assume fully rational and inter-temporal utility maximizing agents, which do not interact directly. The most important model from this category is the rank model, which is fully disconnected (agents can be simulated in isolation). The rest of the models from this category are the stock 
model and order model, in which indirect interaction between agents takes place. Due to the assumption of full rationality, these models are very useful as reference cases, therefore support was added (with a focus on the rank model).

In the interaction-based models, the decision to adopt relies on the interaction and information exchange between a network of agents. Usually, the interacting agents use rather simple decision rules. The simplest models from this category (from an analytical point of view) are the ones based on statistical mechanics ${ }^{(21)}$ and resemble the Ising model. Various theoretical results have been obtained by these methods ${ }^{(21)}{ }^{(23)}$, therefore support was added.

Usually, interaction models consider just the demand side and ignore supply. In the the case of emergent technologies, demand-only models prove to be insufficient, as the decision of the supplier to switch to producing the innovated products and the limited supply have a strong effect on the diffusion process. For this purpose, models both for consumers and suppliers were considered necessary.

After the analysis, we concluded that: the framework should be multi-agent based and accommodate micro models, both connected and disconnected, facilitate network interactions between agents and consider the supply side as well.

\subsection{Simulation design patterns}

We analyzed the previously selected models and various simulations in which these models were used in order to discover similarities and common simulation design patterns. We then decided how to use these to design an efficient and coherent simulation framework. The significant points were the following:

- $\quad$ most simulations combine data and models from various sources $\rightarrow$ we designed a distributed simulation infrastructure in which simulation modules are combined in the main simulation

- for a realistic simulation, quite a large number of such modules are needed $\rightarrow$ we designed a service-oriented centralized data/model management system

- data acquisition takes a lot of time $\rightarrow$ the management system was designed so that previously entered data is available to all framework users, independent of location

- almost all aspects considered in the simulation and the result itself vary with time and this evolution is what drives adoption $\rightarrow$ we considered the base element of the simulation to be a service function, a special kind of time series; all simulation modules are represented by these service functions, which can be created in the simulation or received from from remote modules (hence the name service)

- a single-product market with a (large) group of consumers and sometimes a (small) group of suppliers is usually considered; the interaction between consumers and between suppliers is sometimes taken into account; interaction networks are usually the standard ones (grids, small world networks and random graphs) $\rightarrow$ we considered a standard market composed of one product in a number of variants, characterized by attributes represented as service functions, a network of consumers and a network of suppliers; we pre-defined all standard network topologies

\section{The framework}

\subsection{Infrastructure}

A distributed infrastructure was implemented in order to streamline the simulation process. By using a multi-agent infrastructure, a virtual database of "simulation parts" was created. These parts can then be easily accessed and combined inside the main simulation. To accomplish this, we defined a class of service providing agents. Examples of such services are cost forecasts (e.g. electricity cost) or technological characteristics (e.g. fuel availability and range for a FCV). The results are then sent to the main simulation in the form of service functions which, among other features, carry the time range the provided service is valid in and the numerical values in time-series format. We believe these service functions to be a 


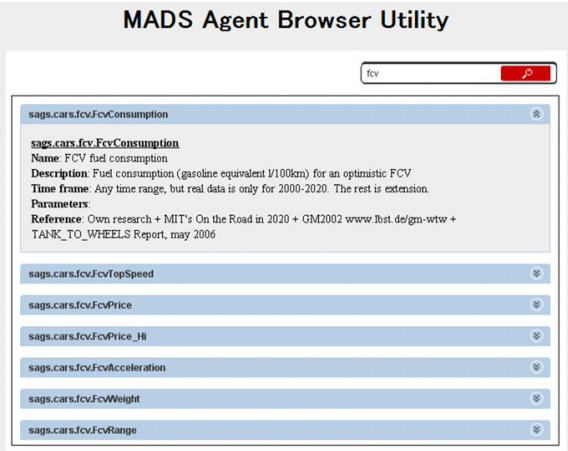

Fig. 1 The MADS Agent Browser Utility web interface is used to query the (usually) remote agent database. Various information regarding the agents' functionality (e.g. description, measurement unit or literature reference), entered at design time, is displayed. MADS is designed such that entering this information is compulsory.

unique feature of out framework.

By designing all the framework in terms of time series rather than individual variables, the dynamic nature of innovations is naturally expressed. Time series are not limited to numeric values (e.g. prices, efficiencies) but include consumer networks, GIS maps and so on. Simulations written in this way become more realistic, as the evolution of all the aspects considered in the simulation is automatically included.

By making this encapsulation possible, the framework enables the user to write a modular simulation, where well defined and well documented modules are created and combined. With time, a searchable agent database is created, leading to easy code maintenance and reuse.

Due to the distributed nature, any user, as long as connected to a central agent server, can use the services provided by the available agents, thus enabling collaboration. Agents can reside on any computer connected to the main server, and the search is conducted through a web interface (Fig.1). An illustration of the process can be seen in Fig.2. The infrastructure was implemented in the JAVA language and uses $\mathrm{JADE}^{(12)}$ for communication and agent management.

\subsection{Simulation development}

The structure of the framework determines the steps one has to take and the data and parameters one needs to find to implement the simulation. This means the framework helps structuring the research and data acquisition process by highlighting what operations one has to do next and what the order of these operations should be. To make the simulation design as simple as possible, depending on the type of simulation, the presence of absence of inter-agent communication etc., different design patterns are provided. In the following, the complex interaction pattern, which was used for studying the FCV diffusion process, will be presented. We will start with a brief outline of the design process. More detailed explanations will be given in the next section, in the context of designing the FCV simulation.

The steps required to implement the complex interaction pattern are the following:

(1) define the product (relevant characteristics)

(2) define the consumer (relevant characteristics + utility function)

(3) define the supplier (relevant characteristics + marketing strategy)

(4) find necessary data for the time evolution of all considered characteristics (product, suppliers, consumers) and encapsulate it into agents

(5) decide the simulation time range

(6) set the initial conditions 


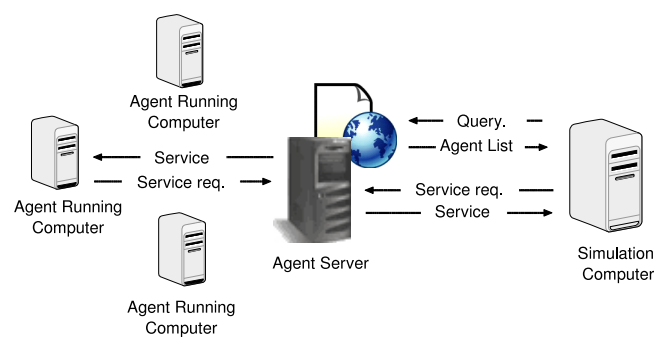

Fig. 2 Layout of the infrastructure. The user looks up agents using the web interface and uses them in the main simulation. The main simulation makes a service request to the agent server, which may either respond directly, in the case the required agent runs locally on the server, or place an additional request to the computer the agent is running on.

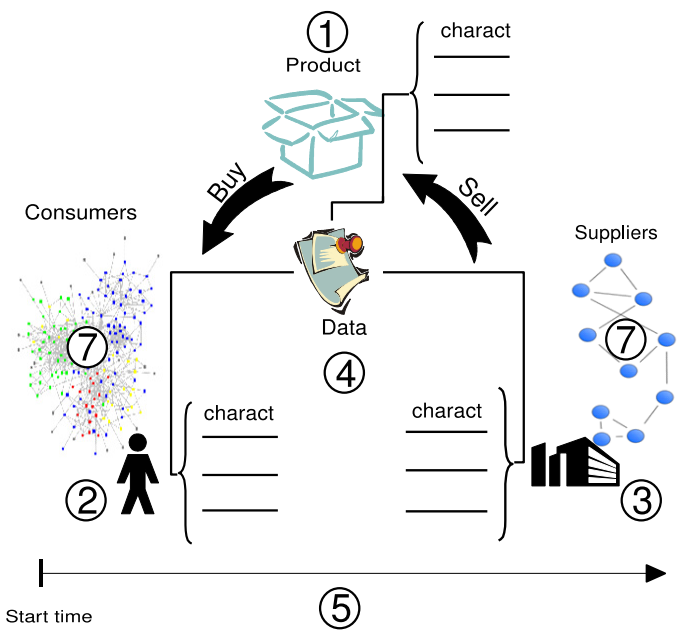

Fig. 3 Complex interaction simulation pattern

(7) set the interaction network

(8) run the simulation

\section{FCV diffusion study}

\subsection{Model \& Implementation}

We followed the above mentioned steps in order to implement the FCV diffusion simulation in the proposed framework. From the simulation models available in MADS, the most suitable was the complex interaction pattern. The underlying diffusion mechanism is therefore the following: a group of four suppliers, representing the three major vehicle types plus the hydrogen refueling infrastructure, compete to sell their products to a large group of consumers. Consumers choose according to vehicle attributes weighted by their personal preferences, while partially influenced by their entourage. Suppliers adapt their production in order to closely match the demand.

We chose to focus the simulation on the US personal car segment, mainly due to data availability and previous research in modeling consumer behavior for the vehicle market.

4.1.1. The product The first step in implementing the simulation is to define the product whose diffusion is going to be studied. Only one generic product can be used in MADS, but with unlimited variants. In this case, the product is a car and its variants are gasoline, gasolinehybrid and fuel cell. More vehicle variants, like diesels, diesel-hybrids or electric vehicles are currently being analyzed. 
A product is defined by specifying its characteristics and production data. While the former is always necessary the latter must be defined just in case a special kind of supplier, which in this case will not be used, is chosen.

The characteristics include all relevant attributes a consumer might consider when computing the product's utility. While the attributes will be the same for all product variants, their values will vary from variant to variant and with time. In this case, the considered characteristics are: vehicle price, fuel cost, vehicle range, acceleration, fuel availability and top speed.

4.1.2. Consumer utility The adoption of a car depends on a number of characteristics. While modeling the adoption we use a "hedonic" approach, meaning that the utility that can be derived from a car comes from its attributes and one can get the overall utility by adding the utility of each individual attribute. Practically, this means that we are going to assign to each attribute (e.g. acceleration, fuel economy) an amount of money a consumer is willing to spend to have it, then add these values to the price of the vehicle and obtain the "real price". The smaller the real price, the bigger the utility. This would translate to, for example, a cheaper gasoline vehicle with poor fuel economy being actually more expensive (in "real price") than a more efficient but more costly hybrid vehicle.

Valuating the attributes of a product is probably the hardest part from the simulation design process. Fortunately, in the case of vehicles, several models can be found in literature: a regression based study by Adamson ${ }^{(4),(5)}$, a revealed-preference based study by Greene and Chin $^{(24)}$, a declared-preference study by Tompkins ${ }^{(25)}$ and the AVID model ${ }^{(26)}$ based on the last two.

Adamson establishes the values of the individual attributes by regression, and does this for a number of years. The values vary from year to year in a non-monotonic way, making the task of deriving a future trend almost impossible. The model was also intended just for the second niche buyers (early buyers here). We therefore chose not to use this model. We decided not to use Green and Chin's or Tompkins' models either, because of the rather significant differences between the two. Our choice was the AVID model because it discriminates between different types of buyers, provides a series of coefficients and the way they evolve in time and seems more reliable as it is based on surveys and not on regression.

As recommended by the AVID model, we discriminate between three category of consumers: early adopters, early buyers and majority buyers. The early adopters and early buyers form the niche market. This is the first market an innovation has to pass through so that it matures and reaches the general public.

Early adopters are high income individuals who value the novelty, the innovation itself. They are not so sensitive to price and are willing to adopt a sufficiently appealing innovation. They are the first category of consumers a new product is targeted to. Early buyers are sufficiently-high income individuals which are not so interested in novelty, but willing to pay a premium for increased performance and efficiency. It is considered that they are not constrained by money. The majority buyers are the regular buyers, constrained by money. All three categories value each attribute differently.

The considered attributes and the value they represent (expressed in US dollars), as provided by the AVID model, are listed in Table 1.

Table 1 The vehicle attributes and their valuation. Negative value means "smaller is better".

\begin{tabular}{lccc}
\multicolumn{1}{c}{ Attribute } & Unit & Early adopters & Majority \\
\hline Vehicle price & $\$$ & -1 & -1 \\
Fuel cost & $\$ /$ liter & -2896.8 & -764.4 \\
Range & $1 / \mathrm{km}$ & -18024 & -186007 \\
Acceleration & sec $0-100 \mathrm{kph}$ & -1000 & -217 \\
Fuel availability & $1 /$ ratio to gas & -75.7 & -17.7 \\
Top speed & $\mathrm{km} / \mathrm{h}$ & 69.5 & 15 \\
Familiarity & $\$ /$ ratio & 0 & 1500 \\
\hline
\end{tabular}


For the familiarity term (external interaction term), not present in AVID, the corresponding weight was computed as a residual between the historical path for hybrids between 20002005 and the path obtained without using this term. The decision to adopt was also considered to be related to the consumer's income.

The values for early buyers are considered somewhere between early adopters and majority, and can be computed with the formula:

$$
V_{e b}=f \cdot\left(V_{e a}-V_{m}\right)+V_{m}
$$

where $V_{e b}$ represents the value for early buyers, $V_{e a}$ the one for early adopters, $V_{m}$ the one for the majority and $f$ represents the position between the two. While the values for early buyers and majority buyers remain constant, the ones for early adopters, after some time (in our case 5 years) start a process of exponential decay until they reach the values for the early buyers. This represents the loss in interest for the novelty of the technology, as it gradually becomes popular.

The values presented in Table 1 should be interpreted in relation to the difference between two different vehicle options. For example, the acceleration value of 1000 for early adopters means that an early adopter would pay an extra 1000 dollars for a decrease of 1 second in the acceleration time from 0 to $100 \mathrm{~km} / \mathrm{h}$. Therefore, these values become coefficients by which differences in attributes can be multiplied with to reveal the real value, in money, the difference in attributes amounts to.

From a MADS point of view, all the coefficients have been incorporated into agents, one for each, which would return upon request an object called here a service function that represents the evolution of one particular value through time. As service function arithmetic is supported by MADS, the coefficient-attribute multiplication can be performed directly and remains valid for any point in time. Storing consumer preferences as agents is very convenient, as different behavioral models can be stored in different agent sets and one can very easily switch or make combinations by simply changing the name of the service providing agents in the main simulation.

4.1.3. Suppliers Four suppliers were then defined: gasoline vehicle supplier, hybrid supplier, FCV supplier and hydrogen fuel supplier. The gasoline vehicles supplier was considered to be of virtual type (no supply limit). The hybrids and FCVs suppliers were considered to be simple adaptive type (start with a small number of vehicles and increase production each year if demands exceeds supply). For the fuel supplier, we considered an adaptive infrastructure growth scenario, from (8).

4.1.4. Data Data for the evolution of all characteristics was obtained by interpolating between values for the years 2000, 2006, 2010, 2020 and 2030. For 2000 and 2006, real data was taken. In the case of the gasoline car, the US best selling car Toyota Camry was taken as reference while for the gasoline-hybrid the Toyota Prius was used. In the case of FCVs, since no commercial vehicles are yet on the road, data was obtained via a survey of the characteristics of the existing prototypes. For the rest of the years, data was obtained from forecasts $^{(27),(28)}$. This data, which forms our baseline scenario, is shown in Table 2. Regarding top speed, we considered that an electronic limiter will limit the speed at a maximum value of $180 \mathrm{~km} / \mathrm{h}$. The gasoline price was set according to the forecasts of the US DOE (Department of Energy). The price for hydrogen was computed by interpolating between the current price and DOE's target of achieving a price of $\$ 2 /$ GGE by 2015. In the scenarios in which we include taxes on emissions, we consider an aggressive C02 tax of 20 cents per liter starting with 2010 and increasing with 10 cents per liter every five years. The income of consumers was generated according to the US Census data.

According to the MADS data management system, all gathered information, including consumer characteristics, was encapsulated into agents. An overview of the created agents is shown in Table 3. 
Table 2 Data for the base-line scenario. Gasoline vehicles and gasoline-hybrids are denoted by "G" and "G-H", respectively.

\begin{tabular}{|c|c|c|c|c|c|}
\hline & & 2000 & 2006 & 2010 & 2020 \\
\hline \multirow[t]{3}{*}{ Acceleration (s) } & $\mathbf{G}$ & 9.9 & 9.2 & 9.1 & - \\
\hline & G-H & 14.1 & 10.2 & 8.8 & - \\
\hline & FCV & 12 & 11 & 9.4 & 9.3 \\
\hline \multirow[t]{3}{*}{ Fuel cons. / city (l/100km) } & $\mathbf{G}$ & 10.2 & 10.2 & - & 6.2 \\
\hline & G-H & 4.52 & 3.92 & - & 3.75 \\
\hline & FCV & 3.5 & - & - & 2.5 \\
\hline \multirow[t]{3}{*}{ Fuel cons. / highway (l/100km) } & G & 7.3 & 7.3 & - & 4.51 \\
\hline & G-H & 5.22 & 4.61 & - & 2.79 \\
\hline & FCV & 3.5 & - & - & 2.5 \\
\hline \multirow[t]{3}{*}{ Range / city (km) } & G & 985 & 985 & - & 1128 \\
\hline & G-H & 994 & 1147 & - & 1185 \\
\hline & FCV & 300 & 400 & - & 532 \\
\hline \multirow[t]{3}{*}{ Range / highway (km) } & G & 952 & 952 & - & 1549 \\
\hline & G-H & 860 & 975 & - & 1609 \\
\hline & FCV & 300 & 400 & - & 532 \\
\hline \multirow[t]{3}{*}{ Top speed $(\mathbf{k m} / \mathbf{h})$} & $\mathbf{G}$ & 177 & 180 & 180 & 180 \\
\hline & G-H & 160 & 180 & 180 & 180 \\
\hline & FCV & 140 & 150 & - & 180 \\
\hline \multirow[t]{3}{*}{ Price $(\$)$} & $\mathbf{G}$ & 20300 & 19450 & - & 19400 \\
\hline & G-H & 20000 & 21500 & - & 21100 \\
\hline & FCV & 1400000 & 1000000 & - & 22100 \\
\hline Gasoline price $(\$ / \mathbf{l})$ & & 0.36 & 0.62 & 0.63 & 0.76 \\
\hline Hydrogen price (\$/I gas.eq) & & 1.84 & 1.33 & 1.05 & 0.54 \\
\hline
\end{tabular}

4.1.5. Network topology Regarding the network topologies, for consumers we considered that a small world network would be best suited. For suppliers, as we considered only generic representatives, no network was considered.

4.1.6. Time range and initial conditions Finally, we set the initial conditions. The initial segment size (year 2000) was considered 9 million cars, as indicated by the historical data. For the hybrid vehicles the year of introduction was considered 2000 and the initial production set according to history data to 10,000 vehicles. For the fuel cell vehicles, considering the producers' expectations, the year 2015 was considered to be the initial mass commercialization date. The initial number of vehicles was set to 100,000, a large enough volume to ensure some economies of scale and reduce the production price to an accessible level. A number of 5000 agents, representing the consumers, were used for the simulation. According to the established segment size, each agent represents in fact a group of 1800 similar households. According to Toyota's predictions, the size of the early adopter's group was set to $3 \%$. We followed the suggestion of AVID and set the size of the early buyers' group to $12 \%$, so that the whole early group make $15 \%$ of the market. It was considered that the average vehicle replacement time is 4 years, which is in agreement with the statistical data.

Table 3 List of created agents

\begin{tabular}{|c|c|c|}
\hline Fuel Price & Vehicle attributes & Consumer preferences \\
\hline \multirow{3}{*}{$\begin{array}{l}\text { gasoline price } \\
\text { hydrogen price }\end{array}$} & Gasoline $\quad$ Gasoline-hybrid FCV & Early adopters $\quad$ Early buyers Majority \\
\hline & acceleration $(\mathrm{G}, \mathrm{G}-\mathrm{H}, \mathrm{FCV})$ & sens. to accel. (EA, EB, M) \\
\hline & fuel cons. city (G, G-H, FCV) & sens. to fuel cons. (EA, EB, M) \\
\hline Other & fuel cons. highway (G, G-H, FCV) & sens. to range $(\mathrm{EA}, \mathrm{EB}, \mathrm{M})$ \\
\hline \multirow{4}{*}{$\begin{array}{c}\mathrm{CO}_{2} \text { tax } \\
\text { income generator }\end{array}$} & range city $(\mathrm{G}, \mathrm{G}-\mathrm{H}, \mathrm{FCV})$ & sens. to speed (EA, EB, M) \\
\hline & range highway $(\mathrm{G}, \mathrm{G}-\mathrm{H}, \mathrm{FCV})$ & sens. to price (EA, EB, M) \\
\hline & top speed (G, G-H, FCV) & sens. to fuel availability (EA, EB, M) \\
\hline & price $(\mathrm{G}, \mathrm{G}-\mathrm{H}, \mathrm{FCV})$ & sens. to familiarity (EA, EB, M) \\
\hline
\end{tabular}

\subsection{Results}

To study various paths to adoption, two key parameters were varied: the exogenous growth of hydrogen-enabled gasoline stations and the presence of an emission tax. Both parameters represent government intervention using the two most important levers: taxes and 
strategic investments. The results were grouped into four different scenarios: FI-TAX (fast infrastructure growth and $\mathrm{CO}_{2}$ tax), FI-NOTAX (fast infrastructure growth but no $\mathrm{CO}_{2} \operatorname{tax}$ ), SI-TAX (slow infrastructure growth and $\mathrm{CO}_{2}$ tax) and SI-NOTAX (slow infrastructure growth but $\mathrm{CO}_{2}$ tax). The obtained diffusion paths are shown in Fig.4 and Fig.5.

The results suggest that diffusion will not happen without external investments in infrastructure. It seems performance alone cannot solve the chicken-and-egg problem. For a fast diffusion, a good choice of the number of hydrogen stations seems to be around $1 \%$ by 2015 , possibly generated by investments in new hydrogen stations of about $0.1 \%$ each year, starting from 2006. This should make the technology attractive enough for the niche market. After this important point is passed and fuel cell vehicles begin to enter the mass market, a self-reinforcing process leads to an exponentially fast infrastructure growth.
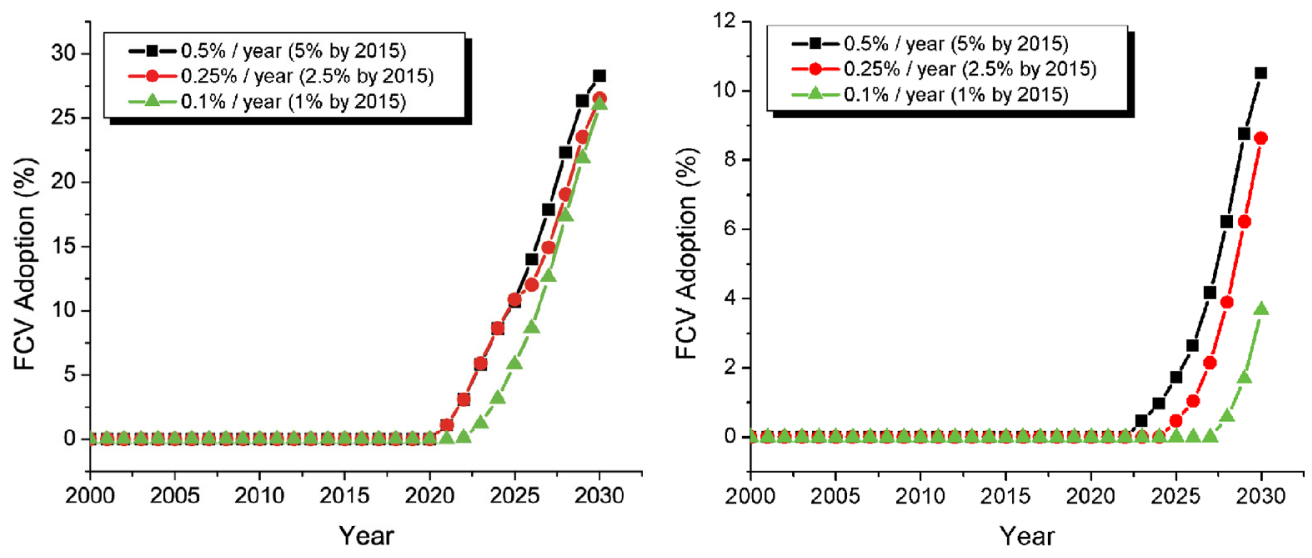

Fig. 4 FCV diffusion trajectories for FI-TAX (left) and FI-NOTAX (right) scenarios
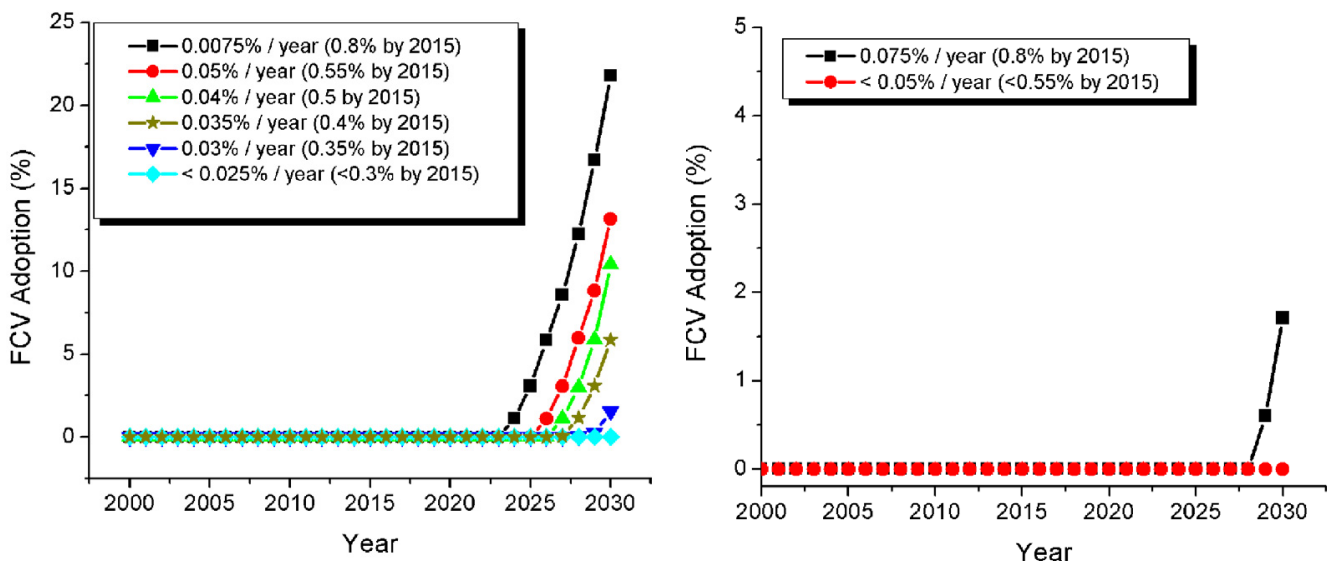

Fig. 5 FCV diffusion trajectories for SI-TAX (left) and SI-NOTAX (right) scenarios

As discussed in the introduction, this simulation technique cannot provide very accurate results, since most externalities are ignored. However, if these results are close enough, using the fact that our utility function includes various aspects not accounted for in the traditional simulation technique, sensitivity analysis with respect to these aspects can be performed.

We computed the sensitivity with respect to price, fuel efficiency, range and acceleration by varying each parameter while keeping the rest fixed, and comparing the adoption percent achieved by the year 2030. The results are shown in Fig.6. It is interesting to see that the sensitivity to acceleration is much larger that the one to range or fuel efficiency. This is 


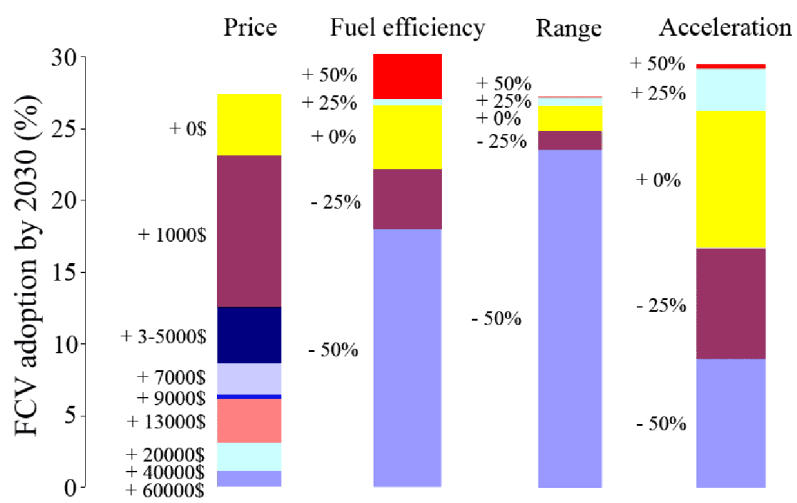

Fig. 6 Sensitivity to price, fuel efficiency, range and acceleration

probably an effect of the high value of the corresponding AVID coefficient for early adopters, coupled with their property of spear-heading the adoption process.

As argued at the beginning of this section, for a trustworthy sensitivity analysis, fairly accurate diffusion trajectories are required. For this purpose, a comparison was made with the literature for both FCVs and gasoline-hybrids. Although our purpose was to simulate the adoption of fuel cell vehicles, the most significant test is the one for the hybrids. This is because while present forecasts regarding the future of fuel cell vehicles depend on so many uncertain parameters, the hybrids are already present in the market.

Comparison with other hybrid-gasoline predictions from literature are shown in Fig.7. Our results match the historical data almost perfectly and for the next decade are somewhere in the middle of the current forecasts. For 2006 - 2010, our results seem to follow the general prediction trend. After that, for 2011 - 2015 they steer towards the pessimistic side. The peak of the diffusion of hybrid-gasoline vehicles seems to be at around 2015, after which they begin to lose to FCVs. This behavior is caused by the fact that at this time the hybrid-gasoline vehicles did not reach the majority yet and are currently owned by early adopters and early buyers which will slowly abandon them in favor of the FCVs.

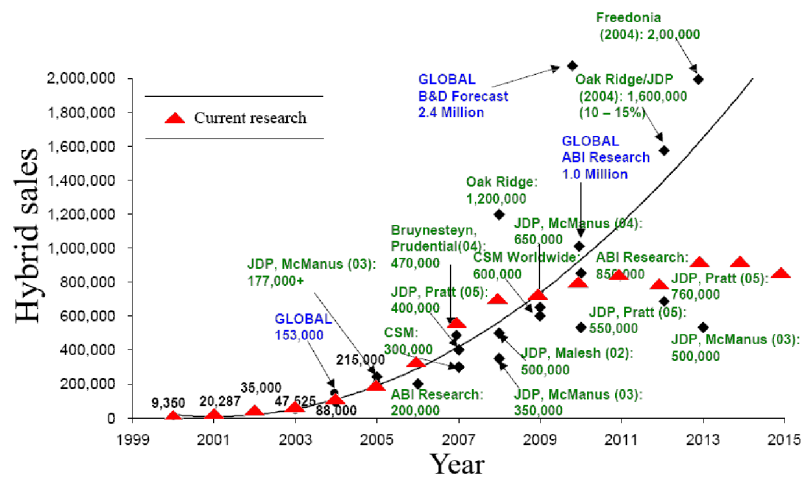

Fig. 7 Comparison between our results for the gasoline-hybrid adoption and literature

Regarding the diffusion of FCVs, we are close the results found in literature. Our scenarios are more optimistic than IEA's ${ }^{(29)}$ but more pessimistic than (30).

\section{Conclusions}

We have developed a new software framework for simulating the diffusion of innovations and we applied it to analyze one of the most important aspects in the transition to a green 
economy: the diffusion of FCVs. Simulating this complex problem confirmed the utility of this framework.

The proposed framework enabled us to develop a fairly short and comprehensible simulation. A lot of data regarding vehicle attributes, fuel prices, consumer utility weights and so on had to be gathered. All gathered data was added to the agent database, which currently includes more than 100 service providing agents. The benefits are four-fold. For one, we have a growing data repository where information is well ordered and can be retrieved and called at any time in any further simulation. Second, any user of this framework can have direct access to this data, regardless of location, thus reducing most of the simulation design process. Third, any correction or update to the data has to be made in just one place and will instantly reflect in all simulations, as every time a simulation is run updated data is requested from the server. Finally, the size of the simulation itself was greatly reduced due to the data being remotely stored.

The results of the simulation were close to our expectations. Both the diffusion path of hybrids and the one of FCVs are similar to those forecasted by other researchers. On the other hand, by working at the actor level, we were able to see how this diffusion will be influenced by not only political decisions but also by technological development and other factors. In particular, the sensitivity analysis revealed the importance of the acceleration for the diffusion of FCVs. Although this could be seen directly from the AVID model, the analysis shows that not falling under the baseline is much more important than trying to go above it and, if resources have to be committed in order for something to be improved above the baseline, then they should be allocated towards improving fuel efficiency.

There are still some open issues to be dealt with. Inside our model, we used a "fuel availability" term. This represents the ratio of hydrogen fuel availability as compared to standard gasoline. Infrastructure development is seen as an increase of this percentage. The assumption is that a number of "well placed" stations are built towards achieving the target coverage. We are currently developing a GIS-enabled infrastructure growth model, in which the infrastructure supplier will optimize a cost function and decide how many stations should be built and where should them be placed on the map. Consumers will also be placed on this map and actually compute the fuel availability ratio over a specified radius.

\section{References}

( 1 ) ETSAP (Energy Technology Systems Analysis Programme), MARKAL (Market Allocation), http://www.etsap.org/markal.

( 2 ) Energy Information Administration, Integrating Module of the National Energy Modeling System: Model Documentation. DOE/EIA-M057 (2001)

( 3 ) S. Messner, L. Schrattenholzer, Message-macro: Linking an energy supply model with a macroeconomic model and solving it interactively, Energy 25 (2000) 267-282.

( 4 ) K. A. Adamson, An examination of consumer demand in the secondary niche market for fuel cell vehicles in Europe, International Journal of Hydrogen Energy 28 (2003) $771-780$.

( 5 ) K. A. Adamson, Calculating the price trajectory of adoption of fuel cell vehicles, International Journal of Hydrogen Energy 30 (2005) 341-350.

( 6 ) R. Madlener, M. Wickart, Diffusion of cogeneration in swiss industries: economics, technical change, field of application, and framework conditions, Energy and Environment 15 (2004) 223-238.

( 7 ) M. Schwoon, Optimal initial distribution of hydrogen filling stations, Proceedings of the second HyCARE meeting, Luxenburg, Dec. 2005.

( 8 ) M. Schwoon, Simulating the adoption of fuel cell vehicles, Journal of Evolutionary Economics 16 (2006) 435-472.

( 9 ) MADS, http://nihonbashi.race.u-tokyo.ac.jp/mads/.

(10) The SWARM Simulation System. www.swarm.org 
(11) M. J. North, N. T. Collier, J. R. Vos, Experiences creating three implementations of the REPAST agent modeling toolkit, ACM Transactions on Modeling and Computer Simulation, 16 (2006) 1-25.

(12) F. Bellifemine, A. Poggi, G. Rimassa, JADE - a FIPA-compliant agent framework, in: PAAM99. Proceedings of the Fourth International Conference on the Practical Applications of Intelligent Agents and Multi-agent Technology, 1999, pp. 97-108.

(13) The MADKIT Project (Multi-Agent Development Kit), www.madkit.org.

(14) M. Parker, ASCAPE, http://brook.edu/es/dynamics/models/ascape.

(15) F. Bousquet, I. Bakam, H. Proton, C. L. Page, CORMAS: Common-pool resources and multi-agents systems, Lecture Notes in Artificial Intelligence (Subseries of Lecture Notes in Computer Science) 1416 (1998) 826.

(16) D. Phan, Cognitive Economics, Springer Verlag, 2004, Ch. From Agent-Based Computational Economics towards Cognitive Economics, pp. 71-398.

(17) R. S. Gray, G. Cybenko, D. Kotz, R. A. Peterson, D. Rus, D’Agents: Applications and performance of a mobile-agent system, Software- Practice and Experience 32 (6) (2002) 543-573.

(18) JINI, http://www.jini.org/.

(19) J. A. Davila, M. Uzcategui, Galatea: A multi-agent simulation platform, AMSE Special Issue 20002002 (2000) 5267.

(20) M. Karshenas, P. L. Stoneman, Rank, stock, order, and epidemic effects in the diffusion of new process technologies: an empirical model, Rand Journal of Economics 24 (4) (1993) 503-528.

(21) H. P. Young, Diffusion in social networks, Tech. Rep. 2, Brookings Institution - Working Papers (1999).

(22) W. A. Brock., S. N. Durlauf, Discrete choice with social interactions, The Review of Economic Studies 68 (2) (2001) 235-260.

(23) L. E. Blume, The statistical mechanics of strategic interaction, Games and Economic Behavior 5 (3) (1993) 387-424.

(24) D. Greene, S. Chin, Alternative fuels and vehicles (AVF) model changes, Center for Transportation Analysis, Oak Ridge, Tenn. (2000).

(25) M. Tompkins et all, Determinants of alternative fuel vehicle choice in the continental united states, Tech. rep., Transportation Research Record 1641: 130-138 (1998).

(26) D. Santini, A. Vyas, Suggestions for a new vehicle choice model simulating advanced vehicles introduction decisions (AVID): Structure and coefficients, Argonne National Laboratory (2005).

(27) M. A. Weiss, J. B. Heywood, E. M. Drake, A. Schafe, F. F. Yeung, On the road in 2020 - A life-cycle analysis of new automobile technologies, MIT Energy Laboratory (2000).

(28) EU, Well-to-wheels analysis of future automotive fuels and powertrains in the European context, tank-to-wheels report, European Commission (2006).

(29) IEA, Prospects for hydrogen and fuel cells, 2005, ISBN: 92-64-10957-9.

(30) M. Mintz, S. Folga, J. Gillette, J. Molburg, Hydrogen: On the horizon or just a mirage?, Tech. Rep. 02FCC-155, Argonne National Lab (2002). 